\section{Pitfalls in dengue case}

\section{management}

\section{Siripen Kalayanarooj* and Mukda Vangveeravong}

Queen Sirikit National Institute of Child Health, Department of Medical Services, Ministry of Public Health, Bangkok 10400, Thailand
Received: 28 December, 2020

Accepted: 23 January, 2021

Published: 27 January, 2021

*Corresponding author: Siripen Kalayanarooj, Queen Sirikit National Institute of Child Health, Department of Medical Services, Ministry of Public Health, Bangkok 10400, Thailand, E-mail: siripenk@gmail.com

https://www.peertechz.com

\section{Check for updates}

Dengue is one of the most common mosquito-borne viral diseases affecting both children and adults worldwide, particularly in the countries of Asia and Latin America [1]. An estimated 100-400 million infections occur annually with 50,000 deaths per year [1]. Most symptomatic patients with dengue present with mild non-specific symptoms that do not require hospitalization [1-3]. Patients with mild to moderate dengue severity often have clinical manifestations of poor appetite, nausea, vomiting, abdominal pain, and dehydration $[2,3]$. A number of patients with mild to moderate dengue severity need supportive and symptomatic treatment in the hospital, which mainly comprises intravenous fluid therapy [1-4]. Severe or complicated cases require hospitalization according to the disease because of plasma leakage [Dengue Hemorrhagic Fever (DHF)/Dengue Shock Syndrome (DSS)] and bleeding [1-4]. Close monitoring and hospitalization are required in patients with dengue who have a risk of death based on characteristics including infancy; elderly age; pregnancy; obesity; and the presence of bleeding, prolonged shock, co-infections, and co-morbidities [2-4]. According to the dengue death review in Thailand, there are pitfalls in dengue case management, which lead to prolonged shock and subsequent multiple organs failure and death. The latest review of 140 of 307 dengue deaths (45.6\%) was between 2017 and 2019. The major causes of death were fluid overload (32\%), massive bleeding $(30 \%)$, prolonged shock (22\%) and unusual presentations (16\%) (Unpublished data). However, majority of the expired patients had multiple causes of death as described. These causes of death could be explained as follows:

\section{Delayed detection of plasma leakage}

\section{Resulting in excessive intravenous fluid administration}

o Monitoring the occurrence of plasma leakage among patients with dengue is needed on day 3 of fever onset and afterward. The simple methods for determining plasma leakage in uncomplicated cases include repeating hematocrit and platelet counts, serial monitoring of serum albumin levels, and chest radiography and ultrasonography by experienced personnel. However, chest radiography is not sufficiently sensitive to detect minimal pleural effusion, especially portable chest radiography for both supine and upright views. Thus, it may mislead clinicians in terms of the dengue severity, particularly DHF and DSS.

o Intravenous fluid is usually administered to almost all hospitalized patients daily at the maintenance rate or a higher rate if the patients exhibit signs of dehydration without realizing that they are experiencing plasma leakage. Therefore, patients with severe plasma leakage develop greater pleural effusion/ascites, leading to respiratory distress and subsequent organ failure.

\section{Delayed detection of shock}

Particularly in adults with dengue, leading to a delay in appropriate management

o Patients with DSS usually have good consciousness, even during profound shock. These patients can walk and talk, although they may feel very weak, especially when they do not have a fever. The simple clinical signs that are recommended for all doctors and nurses to be aware of detect shock early among patients with dengue include fainting, giddiness, shortness of breath, abdominal discomfort, postural hypotension, cold and clammy skin, a delayed capillary refill time $>2 \mathrm{~s}$, and a rapid and weak pulse. However, the digital machine for blood pressure monitoring is excessively sensitive such that the machine may measure the blood pressure and heart rate even when patients are in profound shock with a very weak pulse or no palpable pulse. Thus, manual blood pressure monitoring remains an essential 
method for determining shock among patients with dengue.

\section{Delayed detection of significant bleeding}

Resulting in delayed blood transfusion

o Bleeding may not be observed or concealed/internal bleeding may occur, commonly caused by upper Gastrointestinal (GI) bleeding. Patients with prolonged shock, with peptic ulcer disease, and receiving nonsteroidal antiinflammatory drugs or anti-platelet/ anti-coagulant agents are more likely to present with upper GI bleeding from ulcers or gastritis. Other causes of significant bleeding are menstruation and intravascular hemolysis from thalassemia or glucose-6phosphate dehydrogenase deficiency.

o Hematocrit usually increase by less than $5-10 \%$ from baseline in the early stages of dengue due to the occurrence of plasma leakage in patients with DHF or DSS. However, hematocrit may decrease in late stages, particularly among patients with prolonged shock. Blood transfusion is recommended at hematocrit levels of $40-45 \%$, as the actual hematocrit may be less than $30 \%$ due to the development of plasma leakage, leading to an increase in hematocrit by $30-40 \%$ among patients with DSS. Please note that in other diseases, blood is transfused when hematocrit is less than $30 \%$ or lower, as plasma leakage is not present in other diseases, in contrast to that in patients with DHF or DSS.

\section{Delayed diagnosis in unusual cases (expanded dengue syndrome - EDS)}

Resulting in delayed management of common complications of DHF/DSS including Acidosis, Bleeding, hypoCalcemia, low blood Sugar, and Fluid overload (A, B, C, S, F).

o Blood transfusion is the most important approach for these complications as patients with DSS particularly prolonged shock usually have significant (concealed) GI bleeding. Patients with EDS usually present with high fever and shock, who may be misdiagnosed with septic shock, encephalitis, or encephalopathy. The important clues for identifying EDS are the detection of plasma leakage and bleeding. However, EDS is commonly observed in patients with dengue with co-infections or co-morbidities.

\section{References}

1. WHO (2011) Comprehensive Guidelines for Prevention and Control of Dengue and Dengue Hemorrhagic Fever. New Delhi. WHO Regional Office for Southeast Asia, Revised and Expanded Edition. Link: https://bit.ly/3iMH4Tu

2. Kalayanarooj S, Rothman AL, Srikiatkhachorn A (2017) Case Management of Dengue: Lessons Learned. J Infect Dis 215: S79-S88. Link: https://bit. ly/3iOlw8w

3. Kalayanarooj S, Vangveeravong M, Vatcharasaevee V (2018) Clinical Practice Guidelines for Dengue Case Management (Thai). Queen Sirikit National Institute of Child Health, eighty-year anniversary, Ministry of Public Health: Bangkok, Thailand, 2nd edition.

4. Thanachartwet $V(2017)$ Clinical management of severe dengue in adults (Thai). Faculty of Tropical Medicine, Mahidol University, Beyond Enterprise Company Limited: Bangkok, Thailand, 2017.

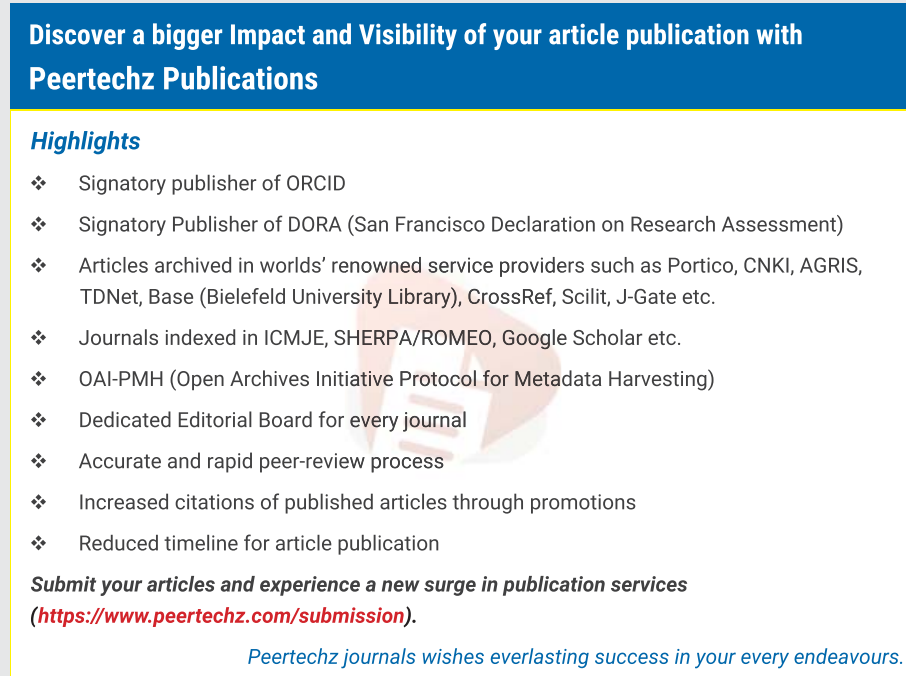

Copyright: @ 2021 Kalayanarooj S, et al. This is an open-access article distributed under the terms of the Creative Commons Attribution License, which permits unrestricted use, distribution, and r eproduction in any medium, provided the original author and source are credited. 